\title{
Controlador Adaptativo PD por Modelo de Referencia para una Mesa Vibratoria Biaxial Basada en el Mecanismo Biela- Manivela
}

\author{
Carlos H. Esparza y Rafael A. Núñez \\ Unidades Tecnológicas de Santander - UTS, Grupo de Investigación en Control Avanzado - GICAV, \\ Calle de los estudiantes No. 9-82, Ciudadela Real de Minas, Bucaramanga-Colombia \\ (e-mail: carlosesfra@gmail.com, ing.rafaeln@gmail.com)
}

Recibido Sep. 6, 2013; Aceptado Oct. 21, 2013; Versión final recibida Nov. 18, 2013

\begin{abstract}
Resumen
Este trabajo presenta la validación de un sistema de control adaptativo por modelo de referencia con ley de control proporcional-derivativo MRAC-PD, que es usado como sistema de control de una mesa vibratoria de dos ejes. La mesa controlada se basa en un mecanismo biela-manivela utilizada para reproducir movimientos sísmicos sobre modelos a escala de estructuras civiles. El controlador MRAC-PD se diseñó en base a la regla MIT de optimización como mecanismo de adaptación de los parámetros proporcional y derivativo. Los resultados fueron mejores que los obtenidos con controladores MRAC con uno y dos parámetros de adaptación proporcionales, controladores adaptativos con predictor Smith para compensar el error introducido por los retardos de los sistemas, y un controlador Fuzzy. La validación de los sistemas de control se implementa en una plataforma hardware de 32 bits de Microchip y se accede de forma remota a través de la red nacional RENATA.
\end{abstract}

Palabras clave: control adaptativo, controlador MRAC-PD, predictor Smith, mesa vibratoria, biela-manivela

\section{Model Reference Adaptive Controller for Biaxial Shaking Table Based on the Slider-Crank Mechanism}

\begin{abstract}
This work shows the validation of a model reference adaptive controller with proportional-derivative control law MRAC-PD. This model is designed as a control system of a shaking table with two axis, based on a slider-crank mechanism, and it is used to generate earthquakes on scale models of civilian structures. The MRAC-PD design was based on the MIT optimization rule as adaptation mechanism for the proportional and derivative parameters. The results are better than those obtained with MRAC controllers with one and two proportional adaptation parameters, adaptive controllers with Smith predictor to compensate the delay errors introduced by the systems and a Fuzzy controller. The validation of the control systems are implemented on a 32 bits hardware platform from Microchip and it is remotely accessed through the national RENATA network.
\end{abstract}

Keywords: adaptive control, MRAC Controller, Smith predictor, shaking table, structural control, slider-crank 


\section{INTRODUCCIÓN}

En el análisis estructural existen diversas técnicas empleadas para evaluar el comportamiento antisísmico de edificaciones, siendo las mesas vibratorias las más empleadas por su aproximación real sobre estructuras a escala o en tamaño real (Seki et al., 2009; Tu et al., 2010). A nivel mundial existen diferentes tipos de mesas vibratorias empleadas para la simulación de terremotos, que dependen del tamaño y peso que pueden soportar, o del número de grados de libertad de desplazamiento. No obstante, en general la mayoría de estas trabajan con actuadores lineales, ya sea eléctricos o hidráulicos, que facilitan el control individual en cada uno de los grados de libertad de la mesa (Yao et al., 2010).

Teniendo en cuenta que el análisis estructural se debe realizar con señales sísmicas patrón, la importancia de los sistemas de control de las mesas vibratorias radica en el alto grado de repetitividad de los experimentos para efectuar los análisis comparativos (Chen et al., 2008). De esta manera, gran parte de los controladores implementados en esta clase de sistemas se basan en técnicas avanzadas como controladores adaptativos, o control robusto y sus diferentes variaciones, especialmente porque esta clase de sistemas acoplados presentan incertidumbre, no linealidad y varianza en el tiempo.

En este caso en particular, la mesa vibratoria se basa en el mecanismo biela-manivela, que genera un desplazamiento lineal en sus dos ejes a partir de una rotación angular de dos motores de inducción trifásicos. Cada motor está acoplado al mecanismo mediante un sistema de poleas y cadenas, y su frecuencia de trabajo es controlada por variadores de velocidad (Esparza et al., 2013). Si bien el sistema de poleas y cadenas disminuyen el torque en los ejes, a su vez disminuye la velocidad de desplazamiento a 220 milímetros por segundo, haciendo que la frecuencia máxima de operación de la mesa sea de $4 \mathrm{~Hz}$ con amplitudes pico a pico de 27.5 milímetros.

Debido a lo anterior, al acoplar todos estos dispositivos y mecanismos que constituyen la mesa en su totalidad, la obtención de un modelo matemático que describa el comportamiento dinámico de la mesa presenta un alto grado de incertidumbre, por lo que un sistema de control adaptativo se presenta como una solución adecuada para obtener alta repetitividad de los experimentos.

En el presente trabajo se plantea la implementación de un sistema de control de posición adaptativo proporcional-derivativo por modelo de referencia MRAC-PD (Landau et al., 2011; Ioacnou, 2000), aplicado a cada uno de los ejes de la mesa vibratoria para generar réplicas del desplazamiento de un sismo en las componentes Norte-Sur y Este-Oeste. El comportamiento de este controlador se valida respecto a cinco controladores diferentes, basados en control Fuzzy(Esparza et al., 2011) adecuado para controlar sistemas no lineales (Mariño et al., 2006), control MRAC proporcional con uno y dos parámetros de adaptación y control MRAC con uno y dos parámetros con predictor Smith respectivamente(Esparza et al., 2013).

Todos los controladores se implementan sobre una plataforma hardware basada en un microcontrolador PIC32 de 32 bits, el cual controla de forma independiente los dos variadores de velocidad de los motores de inducción a través de la variante análoga. Para iniciar los experimentos el microcontrolador debe recibir información del tipo de controlador a implementar, cuáles son los parámetros iniciales del mismo, y la señal que se va a generar en los dos ejes de la mesa vibratoria. La realimentación de la posición del sistema se realiza con dos encoders acoplados a cada eje mediante un sistema piñón cremallera. En la figura 1 se muestra un diagrama de bloques del sistema y en la figura 2 como se encuentran acoplados los sensores de desplazamiento.

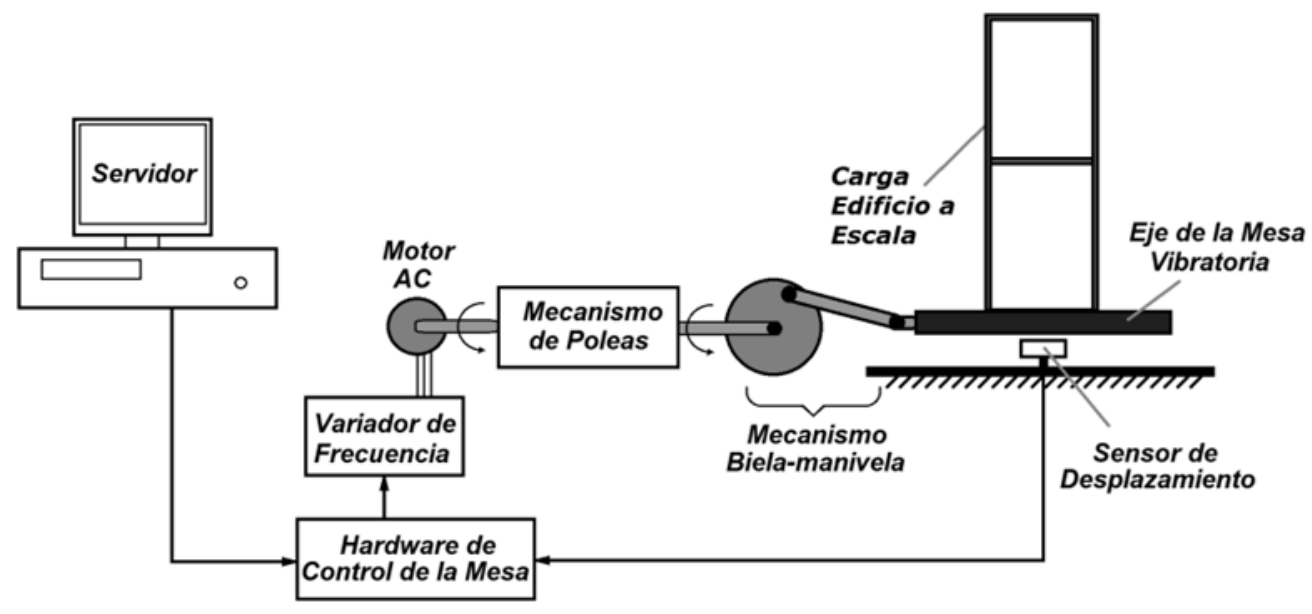

Fig. 1: Conexión del Laboratorio, mesa vibratoria y sistemas antisísmicos. 


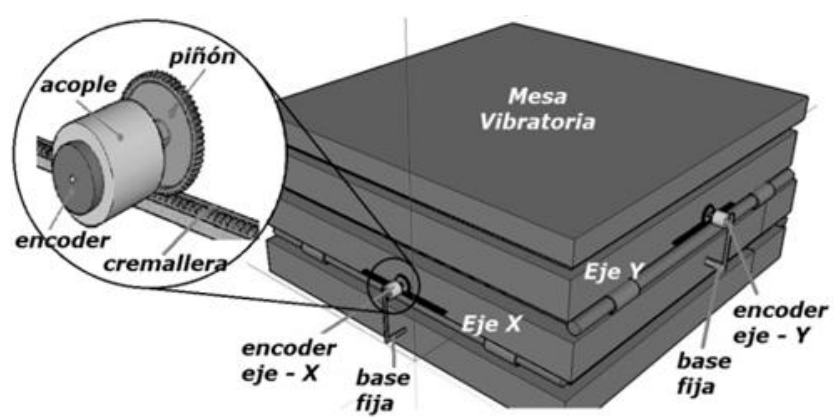

Fig. 2: Ubicación de los encoders en la mesa vibratoria.

Por otra parte, el acceso a los experimentos se realiza de forma remota mediante una aplicación web 2.0 en la cual se puede seleccionar el experimento, realizar varias pruebas, modificar los parámetros iniciales de los controladores, visualizar los resultados de la prueba que se está realizando y descargar los resultados para analizarlos de forma independiente con cualquier software como MATLAB. Para validar los resultados obtenidos con los sistemas de control se implementan las métricas de error de Error Medio Cuadrático Normalizado (Frýza y Hanus, 2003), coeficiente de determinación $R^{2}$ (Montgomery y Runger, 2007)y la métrica de repetitividad dada por el coeficiente de correlación intraclase ICC (McGraw y Wong, 1996) las cuales se explican en la sección de resultados.

Los resultados presentados en este documento forman parte de un proyecto cuyo objetivo es crear un laboratorio remoto de sistemas de control avanzados, el cual pueda ser accesado a través de la Red Nacional Académica de Tecnología Avanzada RENATA, en Colombia, la cual se encuentra conectada a la red CLARA a nivel Latinoamericano. En este laboratorio se implementaran sistemas de control adaptativos y robustos, similar a los laboratorios remotos propuestos por (Aliane et al., 2007) y por (García-Nieto et al., 2007), que permitirá a los alumnos acceder a sistemas complejos como el presentado por (Payá et al., 2007), pero en el que aprovecharán las estructuras de la mesa vibratoria y los modelos a escala de estructuras para desarrollar sistemas antisísmicos.

\section{DESCRIPCIÓN DEL SISTEMA}

En la figura 1 se detalla el diagrama de bloques del laboratorio basado en la mesa vibratoria, la cual se puede desplazar en dos ejes, denominados como eje $X$ y eje $Y$, una distancia máxima de $\pm 10 \mathrm{~cm}$ respecto al a posición central, pero solo de $\pm 6 \mathrm{~cm}$ en el rango determinado de la región lineal, tal como se detalla en la imagen izquierda de la figura 3(Esparza et al., 2013).
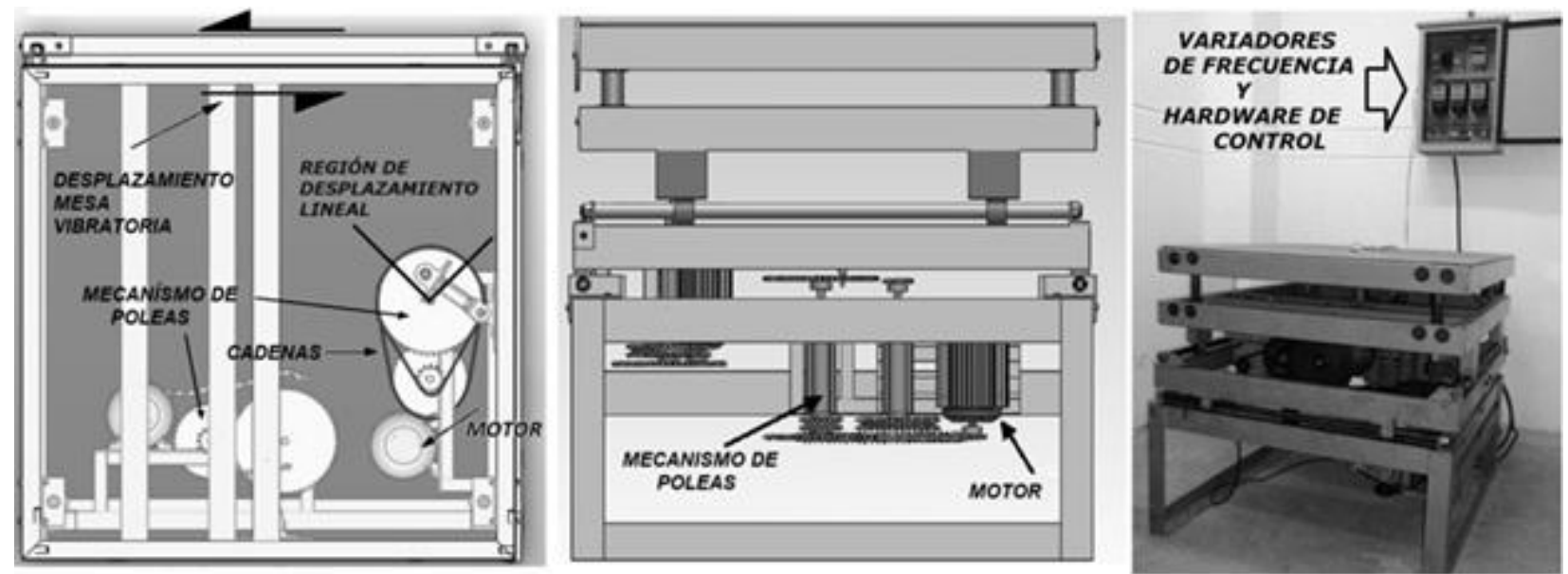

Fig. 3: Izquierda: Mecanismo Biela-Manivela de la mesa, vista superior; Centro: Mecanismo de poleas, vista lateral; Derecha: Fotografía de la Mesa Vibratoria

El funcionamiento del sistema a través de la plataforma web es de la siguiente manera: Primero se selecciona el tipo de sistema de control a implementar en la mesa vibratoria, teniendo como opciones sistema de control Fuzzy, Control adaptativo MRAC con uno y dos parámetros de adaptación y ley de control proporcional, Control adaptativo con uno y dos parámetros de adaptación con predictor Smith y ley de control proporcional, y finalmente sistemas de control adaptativos MRAC con ley de adaptación PD; en segunda instancia se ajustan los parámetros iniciales del controlador seleccionado; posteriormente se selecciona el sismo que se desea generar y se transmite toda la información al microcontrolador encargado de implementar la técnica de control seleccionada; el microcontrolador almacena la información del sistema 
de control y esta es retransmitida a la aplicación web en la que se visualizan las gráficas resultado del experimento. Este proceso se puede realizar varias veces con el fin de validar los controladores y su respuesta ante diferentes valores iniciales de sus parámetros de adaptación.

En cuanto al hardware del sistema, los controladores se implementan en una tarjeta PIC32 Ethernet Starter Kit basada en un microcontrolador de 32 bis del fabricante Microchip. Este se conecta a una tarjeta de expansión con aislamiento digital a través de los cuales se realiza el control de dos variadores de frecuencia SIEMENS G110 mediante su variante análoga. La variante análoga se manipula mediante dos potenciómetros digitales, permitiendo variar la frecuencia de los variadores en el rango de $0 \mathrm{a} 120 \mathrm{~Hz}$, para así controlar la velocidad y el sentido de giro de los motores de inducción trifásicos, según sean los requerimientos de la señal que se va a generar en la mesa vibratoria.

En general la mesa vibratoria pesa aproximadamente $240 \mathrm{Kg}$, y puede soportar un peso adicional de $80 \mathrm{Kg}$ para añadir modelos a escala de edificios u otra estructura. Los experimentos realizados para este artículo se validaron con una carga de $24 \mathrm{Kg}$ correspondiente a un modelo a escala de edificio tipo túnel de dos grados de libertad, y sin carga, obteniéndose un alto grado de repetitividad de las pruebas.

\section{SISTEMAS DE CONTROL IMPLEMENTADOS}

En total en el laboratorio se pueden implementar 6 sistemas de control diferentes, un sistema de control Fuzzy y cinco versiones de control adaptativo por modelo de referencia los cuales se describirán en las siguientes secciones. La ventaja de estos sistemas de control es que no requieren un modelo matemático para ser implementados, sino que requieren un conocimiento general del comportamiento de la planta. No obstante, para validar la respuesta en simulación de los controladores se emplearon dos modelos matemáticos, uno para cada eje.

Los modelos matemáticos de los dos ejes se obtuvieron por medio de técnicas de identificación paramétricas con la toolbox de Identificación de Matlab®. Estos modelos alcanzaron un $91.8 \%$ y $91.97 \%$ de aproximación para los ejes $\mathrm{X}$ y $\mathrm{Y}$ respectivamente. Las funciones de transferencia que describen estos modelos matemáticos se presentan en las ecuaciones 1 y 2 , en las que el retardo se debe al mecanismo de poleas y cadenas de la mesa vibratoria (Esparza et al., 2013).

$\begin{aligned} G_{X}(s) & =\frac{82.739 . e^{-0.075121 s}}{s\left(0.0008484 s^{2}+0.02337 s+1\right)} \\ G_{Y}(s) & =\frac{77.977 . e^{-0.0077277 s}}{s(0.02136 s+1)}\end{aligned}$

\section{SISTEMA DE CONTROL FUZZY SUGENO}

Los controladores Fuzzy se implementaron a partir de dos variables lingüísticas, el error y la razón de cambio del error del desplazamiento de los ejes, y para cada una de ellas se definieron 5 funciones de membresía que subdividen su rango. A su vez, se empleó la técnica Fuzzy Sugeno de promedio ponderado que requiere funciones de membresía simétricas tal y como se muestran en la figura 4.
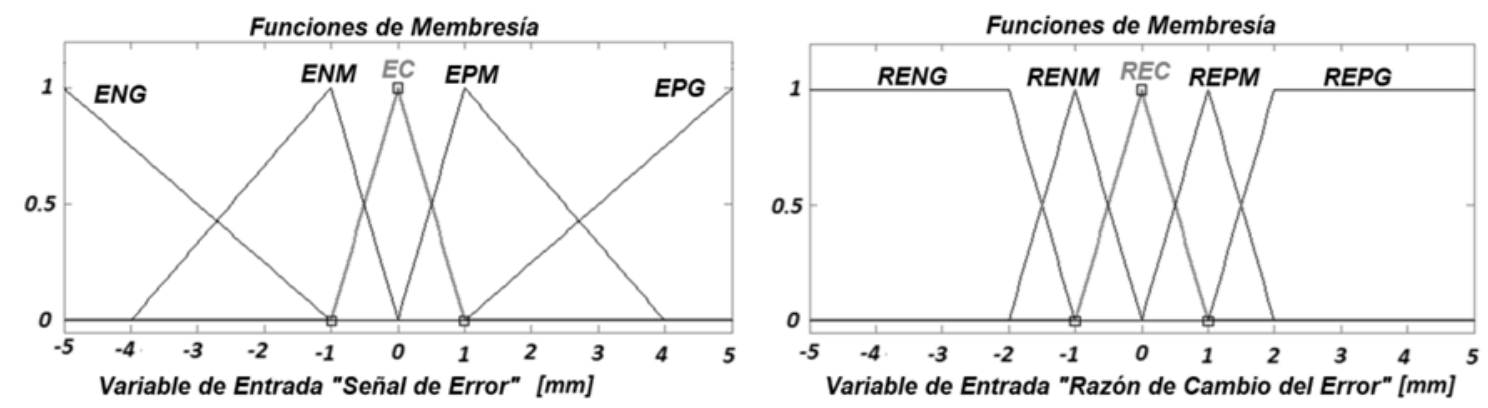

Fig. 4: Izquierda: Funciones de membresía de la señal de Error. Derecha: Funciones de Membresía de la señal razón de cambio del error

En las funciones de membresía de la figura 4, los nombres de cada función corresponden a "Error Negativo Grande", "Error negativo Medio", "Error Cero", "Error Positivo Medio" y "Error Positivo Grande" respectivamente. El proceso de defuzzificación de todas las reglas de salida se calcula a través de la ecuación 3 , correspondiente a la técnica de promedio ponderado, en la que $\omega_{\mathrm{i}}$ corresponde al peso de cada 
regla, $\mathrm{N}$ es el número de reglas que en este caso fueron $25, y \mathrm{z}_{\mathrm{i}}$ es la función de salida que en la topología Sugenodada por la ecuación 4. En la ecuación 4, los avalores a, b y c, son constantes del sistema de control las cuales fueron obtenidas por sintonización.

Output $=\frac{\sum_{i=1}^{N} \omega_{i} z_{i}}{\sum_{i=1}^{N} \omega_{i}}$

$z=a \cdot$ error + b.razón_cambio_error $+c$

\section{CONTROLADORES ADAPTATIVOS POR MODELO DE REFERENCIA - MRAC}

Los sistemas de control adaptativos por modelo de referencia se basan en el diagrama de bloques de la figura 5, los cuales dependiendo de la regla de control implementada modifican los parámetros del controlador MRAC a través del mecanismo de adaptación (Rodríguez y López, 1996).De la figura 5 se aprecia que la entrada de este mecanismo es la diferencia entre la salida real de la planta $Y$ y la salida del modelo de referencia $Y m$, razón por la cual el modelo de referencia corresponde al comportamiento en lazo cerrado de cómo se desea que se comporte la planta ante una entrada $R$.

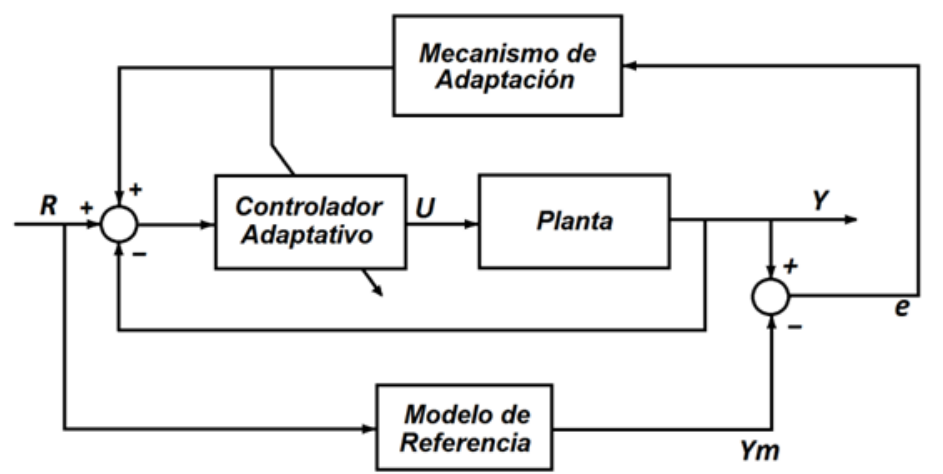

Fig.5: Diagrama de bloques de un Controlador Adaptativo MRAC

Los controladores MRAC planteados se basan en la regla de optimización MIT que minimiza la función de costo de la ecuación 5 , en la que $e$ es la señal de error de la figura 5 y $\theta$ los parámetros de adaptación (Landau et al., 2011). La regla MIT plantea que para minimizar la función Jlos parámetros $\theta$ deben cambiar en la dirección de su gradiente negativo, tal como se plantea en la ecuación 6, en la que $\gamma$ es una constante definida como ganancia de adaptación que determina la rapidez con que se adapta el controlador (Swarnkar et al., 2010; Swarnkar et al., 2011).

$J(\theta)=\frac{1}{2} e^{2}$

$\frac{d \theta}{d t}=-\gamma \frac{\partial J}{\partial \theta}=-\gamma e \frac{\partial e}{\partial \theta}=-\gamma e\left(\frac{\partial Y}{\partial \theta}-\frac{\partial Y m}{\partial \theta}\right)$

Finalmente, la diferencia en los controladores MRAC y la forma en cómo se ajustan sus parámetros radica en la ley de control. En este trabajo se presentan tres sistemas MRAC: el primero con ley de control proporcional y un parámetro de adaptación como se plantea en la ecuación 7 (loacnou, 2000); el segundo con ley de control proporcional y dos parámetros de adaptación como se presenta en la ecuación 8 (Ioacnou, 2000); y el último con ley de control proporcional-diferencial dada por la ecuación 9.

$U=\theta(R-Y)$

$U=\theta_{1} \cdot R-\theta_{2} \cdot Y$

$U=(R-Y)\left(\theta_{p}+\theta_{d} \cdot s \cdot \frac{N}{s+N}\right)$

\section{OBTENCIÓN DE LOS MODELOS DE REFERENCIA}

En el diseño de un controlador MRAC, se debe plantear un modelo de referencia a partir de la respuesta en lazo cerrado del sistema (Duraisamy y Dakshinamurthy, 2010). Partiendo de lo anterior, de la figura 5 se 
tiene la salida $Y$ de la planta como el producto, en términos de Laplace, entre la ley de control $U$ y la planta $G$. La ecuación 10 plantea este resultado para los tres tipos de control planteados en las ecuaciones 7 a 9.

$Y=\theta(R-Y) G ; \quad Y=\left(\theta_{1} \cdot R-\theta_{2} \cdot Y\right) G ; \quad Y=(R-Y)\left(\theta_{p}+\theta_{d} \cdot s \cdot \frac{N}{s+N}\right) G$

Para obtener los modelos de referencia se despeja de la ecuación 10 la relación entre la salida $Y$ y la entrada $R$ del sistema, en las que $G$ corresponden a las ecuaciones 1 y 2 para el eje $\mathrm{X}$ y el eje $Y$ de la mesa respectivamente. Al resolver esto se obtienen las ecuaciones 11,12 y 13 como modelos de referencia para el eje $X$ y las ecuaciones 14, 15 y 16 como modelos de referencia para el eje $Y$. Para el caso de los modelos de referencia de las ecuaciones 13 y 16 , la constante del filtro $N$, obtenida por sintonización, tiene un valor de 6.7699 y 7.7333 respectivamente. Es importante aclarar que en cada uno de los casos se aproximaron las ecuaciones 1 y 2 eliminando el retardo del lazo cerrado y añadiéndolo al final del lazo de realimentación tal como lo propuso Normey en (Normey-Rico y Camacho, 2006).

$$
\begin{aligned}
& \frac{Y_{X}(s)}{R(s)}=\frac{82.739 \theta_{X} e^{-0.075121 s}}{0.0008484 s^{3}+0.02337 s^{2}+s+82.739 \theta_{X}} \\
& \frac{Y_{X}(s)}{R(s)}=\frac{82.739 \theta_{1 X} e^{-0.075121 s}}{0.0008484 s^{3}+0.02337 s^{2}+s+82.739 \theta_{2 X}} \\
& \frac{Y_{X}(s)}{R(s)}=\frac{82.739\left[s\left(\theta_{p X}+6.7699 \theta_{d X}\right)+6.7699 \theta_{p X}\right] e^{-0.075121 s}}{0.0008484 s^{4}+0.029117 s^{3}+1.1582 s^{2}+s\left(6.7699+82.739 \theta_{p X}+560.14 \theta_{d X}\right)+560.14 \theta_{p X}} \\
& \frac{Y_{Y}(s)}{R(s)}=\frac{77.977 \theta_{Y} e^{-0.077277 s}}{0.02136 s^{2}+s+77.977 \theta_{Y}} \\
& \frac{Y_{Y}(s)}{R(s)}=\frac{77.977 \theta_{1} e^{-0.077277 s}}{0.02136 s^{2}+s+77.977 \theta_{2}} \\
& \frac{Y_{Y}(s)}{R(s)}=\frac{77.977\left[s\left(\theta_{p Y}+7.7333 \theta_{d Y}\right)\right] e^{-0.077277 s}}{0.021358 s^{3}+1.1652 s^{2}+s\left(7.7333+77.977 \theta_{p Y}+603.02 \theta_{d Y}\right)+603.02 \theta_{p Y}}
\end{aligned}
$$

Finalmente los parámetros $\theta$ de los modelos de referencia son sintonizados para que la respuesta de la planta sea ideal desde el punto de vista de tiempo de respuesta y sobre impulso del sistema. La tabla 1 presenta los valores obtenidos por sintonización.

Tabla 1: Valores Iniciales de los Parámetros $\theta$ de los controladores MRAC

\begin{tabular}{|l|c|c|}
\hline \multicolumn{1}{|c|}{ Controlador MRAC } & Parámetro 1 & Parámetro 2 \\
\hline Eje X, un parámetro $\theta$ proporcional & $\theta_{X}=0.05$ & -- \\
\hline Eje X, dos parámetros $\theta$ proporcionales & $\theta_{1 X}=0.08$ & $\theta_{2 X}=0.08$ \\
\hline Eje X, dos parámetros $\theta$ PD & $\theta_{p X}=0.067137$ & $\theta_{d X}=0.003456$ \\
\hline Eje Y, un parámetro $\theta$ proporcional & $\theta_{Y}=0.1$ & -- \\
\hline Eje Y, dos parámetros $\theta$ proporcionales & $\theta_{1 Y}=0.05$ & $\theta_{2 Y}=0.05$ \\
\hline Eje Y, dos parámetros $\theta$ PD & $\theta_{p Y}=0.0742884$ & $\theta_{d Y}=0.0060658$ \\
\hline
\end{tabular}

\section{DISEÑO DEL CONTROLADOR MRAC CON UN PARÁMETRO DE ADAPTACIÓN PROPORCIONAL}

Una vez planteados los modelos de referencia se procede a obtener los modelos que describen el comportamiento de los parámetros de adaptación, tomando como punto de partida la ecuación 6 . En esta se desprecia la derivada parcial de la salida del modelo de referencia debido a que este es constante en el tiempo, obteniendo la relación de la ecuación 17.

$\frac{\partial e}{\partial \theta}=\left(\frac{\partial Y}{\partial \theta}-\frac{\partial Y m}{\partial \theta}\right)=\frac{\partial Y}{\partial \theta}$

Al reemplazar las ecuaciones 11 y 14 en la ecuación 17 se tiene (18) y (19).Resolviendo estas derivadas parciales se pueden aplicar nuevamente a la ecuación 6 para determinar cómo es el comportamiento del parámetro de adaptación en el tiempo tal y como se plantea en las ecuaciones 20 y 21 . Cabe resaltar que al 
final los parámetros $\theta$ que quedan en la funcioón de transferencia se toman de los valores iniciales dados por la tabla 1.

$\frac{\partial e}{\partial \theta}=\frac{\partial\left(\frac{\theta_{X} 82.739 e^{-0.075121 s}}{s\left(0.0008484 s^{2}+0.02337 s+1\right)+\theta_{X} 82.739} \cdot R(s)\right)}{\partial \theta_{X}}$

$\frac{\partial e}{\partial \theta}=\frac{\partial\left(\frac{\theta_{Y} 77.977 e^{-0.077277 s}}{s(0.02136 s+1)+\theta_{Y} 77.977} \cdot R(s)\right)}{\partial \theta_{Y}}$

$\theta_{X}=-\frac{\gamma \cdot e \cdot(R(s)-Y(s))}{s}\left(\frac{82.739 e^{-0.075121 s}}{0.00084 s^{3}+0.023 s^{2}+s+4.137}\right)$

$\theta_{Y}=-\frac{\gamma \cdot e \cdot(R(s)-Y(s))}{s}\left(\frac{77.977 e^{-0.077277 s}}{0.021 s^{2}+s+3.89}\right)$

Finalmente, a partir de las ecuaciones 20 y 21 , se obtiene el diagrama de bloques de la figura 6 que describe el comportamiento del controlador MRAC planteado, en el que el bloque denominado Modelo $\theta$ corresponde a las funciones de transferencia de las ecuaciones 20 y 21 para el controlador del eje $\mathrm{X}$ y del eje $\mathrm{Y}$ respectivamente.

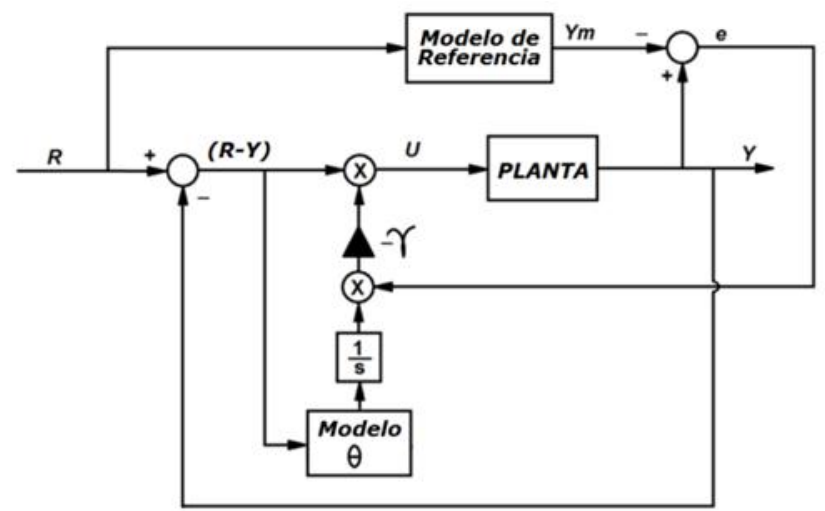

Fig. 6: Diagrama de bloques del Controlador MRAC con un parámetro proporcional

\section{DISEÑO DEL CONTROLADOR MRAC CON DOS PARÁMETROS DE ADAPTACIÓN PROPORCIONAL}

El procedimiento para obtener los modelos que describen el comportamiento de los parámetros de adaptación $\theta_{1}$ y $\theta_{2}$ es el mismo que el realizado en la sección anterior, con la diferencia que la derivada parcial de la ecuación 17 se convierte en un gradiente en las direcciones de cada parámetro $\theta$. Así, al reemplazar las ecuaciones 12 y 15 en la ecuación 17, y posteriormente aplicarlo en la ecuación 6 se obtienen los modelos de las ecuaciones 22 a 25.

$$
\begin{aligned}
\theta_{1 X} & =-\frac{\gamma \cdot e \cdot R(s)}{s}\left(\frac{82.74 e^{-0.075121 s}}{0.0008484 s^{3}+0.02337 s^{2}+s+6.619}\right) \\
\theta_{2 X} & =\frac{\gamma \cdot e \cdot Y(s)}{s}\left(\frac{82.74}{0.0008484 s^{3}+0.02337 s^{2}+s+6.619}\right) \\
\theta_{1 Y} & =-\frac{\gamma \cdot e \cdot R(s)}{s}\left(\frac{77.977 e^{-0.077 s}}{0.02136 s^{2}+s+3.899}\right) \\
\theta_{2 Y} & =\frac{\gamma . e . Y(s)}{s}\left(\frac{77.977}{0.02136 s^{2}+s+3.899}\right)
\end{aligned}
$$

Las ecuaciones 23 y 25 correspondientes al segundo parámetro no incluyen el tiempo muerto, debido a que estos se adaptan de acuerdo a la respuesta de la salida del sistema $Y(s)$ la cual ya incluye el retardo del sistema, a diferencia de las ecuaciones 22 y 24 que dependen de la señal de entrada $R(s)$. Finalmente, el 
diagrama de bloques de la figura 7 presenta el comportamiento del sistema de control MRAC con dos parámetros, en el que los bloques denominados Modelo $\theta_{1}$ y Modelo $\theta_{2}$ corresponden a las funciones de transferencia de las ecuaciones 22 a 25 de los ejes $X$ y $Y$ respectivamente.

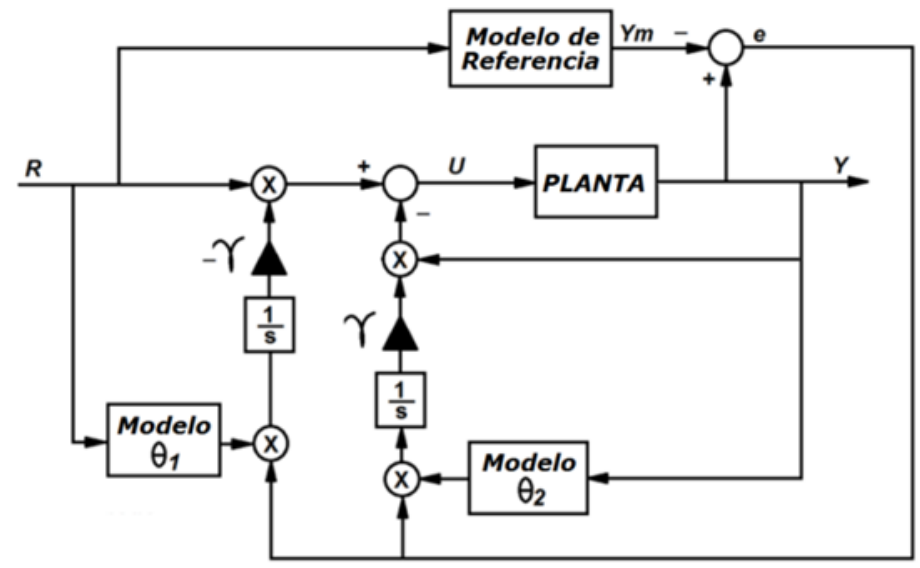

Fig. 7: Diagrama de bloques del Controlador MRAC con dos parámetros proporcionales

\section{DISEÑO DEL CONTROLADOR MRAC PROPORCIONAL-DERIVATIVO PD}

Al reemplazar las ecuaciones 13 y 16 en la ecuación 17, y realizando el mismo procedimiento mencionado anteriormente se obtienen los modelos matemáticos que describen el comportamiento de los parámetros proporcional y derivativo del control MRAC-PD, presentados en las ecuaciones 26 a 29. A su vez el diagrama de bloques que describe el controlador MRAC-PD se presenta en la figura 8.

$$
\begin{aligned}
& \theta_{p X}=-\frac{\gamma \cdot e(R(s)-Y(s))}{s}\left(\frac{82.74 s+560.1}{0.0008484 s^{4}+0.02912 s^{3}+1.158 s^{2}+14.26 s+37.61}\right) \\
& \theta_{d X}=-\gamma \cdot e(R(s)-Y(s))\left(\frac{560.1 s+3792}{0.0008484 s^{5}+0.03486 s^{4}+1.355 s^{3}+22.1 s^{2}+134.2 s+254.6}\right) \\
& \theta_{p Y}=-\frac{\gamma \cdot e(R(s)-Y(s))}{s}\left(\frac{77.98 s+603}{0.02136 s^{3}+1.165 s^{2}+17.18 s+44.8}\right) \\
& \theta_{d Y}=-\gamma \cdot e(R(s)-Y(s))\left(\frac{603 s+4663}{0.02136 s^{4}+1.33 s^{3}+24.62 s^{2}+177.7 s+346.4}\right)
\end{aligned}
$$

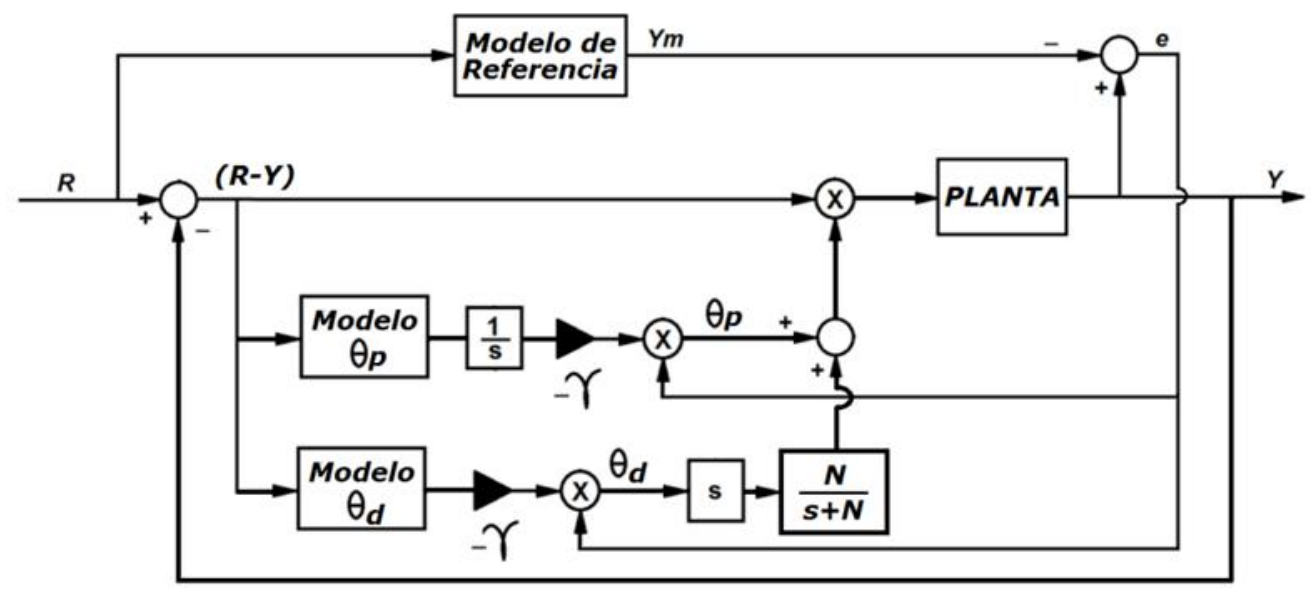

Fig. 8: Diagrama de bloques del Controlador MRAC-PD

\section{APLICACIÓN DEL PREDICTOR SMITH A LOS CONTROLADORES MRAC PROPORCIONALES}

Las técnicas de predictor Smith se plantearon para minimizar los efectos que ocasionan los retardos en una planta con conexión en lazo cerrado. En este trabajo se utilizó la configuración de Modelo de Control Interno (IMC) de la figura 9. 


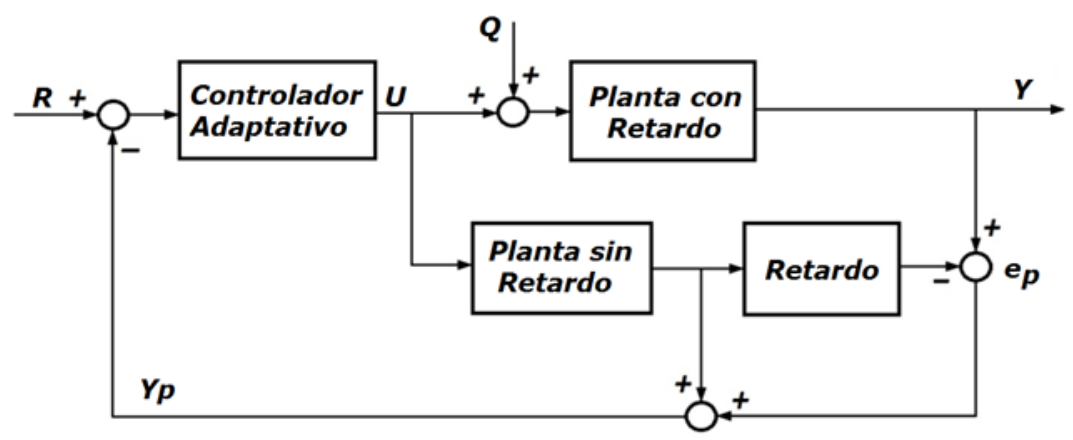

Fig. 9: Estructura de un Controlador con predictor Smith. Representación (IMC)

\section{RESULTADOS}

Una vez diseñados los controladores se convierten al dominio de Z para plantear los algoritmos de control, basados en ecuaciones de diferencias, que se implementan en la plataforma hardware de 32 bits. En total se hicieron 180 pruebas, 60 para experimentos con una señal sísmica, 90 para señales senoidales con diferentes componentes de frecuencia en el rango de $0.4 \mathrm{~Hz}$ a $3 \mathrm{~Hz}$, y 30 señales cuadradas para analizar la respuesta al impulso del sistema con cada controlador. En todos los experimentos se evaluó su repetitividad mediante el coeficiente de determinación $R^{2}$ de la ecuación 30 (Montgomery y Runger, 2007)y el coeficiente de correlación intraclase ICC de la ecuación 31, específicamente el efecto del modelo ICC de dos caminos mezclados(McGraw y Wong, 1996).

Se utilizaron estas métricas debido a que el coeficiente $R^{2}$ permite evaluar la cantidad de variabilidad entre una señal deseada y la señal obtenida, y el coeficiente ICC porque mide el grado de consistencia entre mediciones normalizadas de los datos de prueba. A su vez el ICC evita tendencias sistemáticas presentes en las pruebas experimentales. Este coeficiente es similar al coeficiente de correlación de Pearson, con resultado entre cero y uno, donde un número cercano a la unidad indica una alta repetitividad.

$R^{2}=1-\frac{\sum_{i=1}^{n}\left(x_{i}-y_{i}\right)^{2}}{\sum_{i=1}^{n}\left(x_{i}-\mu_{x i}\right)^{2}}=1-\frac{\operatorname{var}(e)}{\operatorname{var}\left(x_{i}\right)}$

$\operatorname{ICC}(A, 1)=\frac{M S_{R}-M S_{E}}{M S_{R}+(k-1) M S_{E}}$

En la ecuación 30, los términos $x_{i}, y_{i}$ y $\mu_{x i}$ corresponden a la señal deseada, la señal obtenida y la media de la señal deseada respectivamente. En la ecuación 31 los términos $M S_{R}, M S_{E}$ y $k$ corresponden a la media cuadrática entre las filas de las pruebas, el error entre pruebas y el número total de pruebas realizadas. A su vez, en la ecuación 32 se presenta la forma que se obtienen los valores de $M S_{R}$ y $M S_{E}$, los cuales dependen de la varianza de las filas $\sigma_{r}^{2}$, de la varianza del error $\sigma_{e}^{2}$, y la covarianza entre las filas y las columnas $\sigma_{r c}^{2}$. Es importante aclarar que las columnas corresponden a las pruebas y las filas al total de datos de las pruebas.

$M S_{R}=k \sigma_{r}^{2}+\sigma_{e}^{2} \quad ; \quad M S_{E}=\frac{k}{k-1} \sigma_{r c}^{2}+\sigma_{e}^{2}$

El tercer elemento de evaluación usado fue la raíz del error medio cuadrático normalizado (Frýza y Hanus, 2003) que se presenta en la ecuación 33 , con la finalidad de obtener un valor de que tanto error hay entre cada señal generada con la señal deseada.

$N R M S E=\sqrt{\frac{\sum_{i=1}^{n}\left(x_{i}-y_{i}^{\prime}\right)^{2}}{\sum_{i=1}^{n}\left(x_{i}\right)^{2}}}$

De los 60 experimentos con señales sísmicas se realizaron 10 para cada tipo de controlador. El sismo empleado para estos experimentos fue el Imperial Valley ocurrido el 15 de octubre de 1979 en California, Estados Unidos, el cual fue descargado del centro de ingeniería de datos de movimientos fuertes CESMD por sus siglas en inglés. En cuanto a las 90 señales senoidales, se realizaron 3 experimentos por cada tipo de control con las frecuencias de $0.4 \mathrm{~Hz}, 0.7 \mathrm{~Hz}, 1 \mathrm{~Hz}, 2 \mathrm{~Hz}$ y $3 \mathrm{~Hz}$. Todas las señales senoidales tenían una amplitud pico a pico de $40 \mathrm{~mm}$ en cada eje. Por último, se realizaron 5 pruebas para cada tipo de controlador con una señal cuadrada con periodo de 10 segundos y amplitud pico a pico de $60 \mathrm{~mm}$. 
En las tablas 2 y 3 se presentan los resultados de los sistemas de control para la señal sísmica, presentando los valores de NRMSE, $R^{2}$ e ICC, dados todos en porcentaje, para cada uno de los seis sistemas de control implementados en los ejes $X$ y $Y$ de la mesa vibratoria respectivamente.

Tabla 2: Resultados del cálculo de NRMSE, $R^{2}$ e ICC para el eje X con cada controlador. Señal Sísmica

\begin{tabular}{|c|c|c|c|c|c|c|c|c|c|c|c|c|}
\hline \multirow[t]{2}{*}{ Test } & \multicolumn{2}{|c|}{ Fuzzy } & \multicolumn{2}{|c|}{$\begin{array}{l}\text { MRAC } \\
1 \text { Parám. }\end{array}$} & \multicolumn{2}{|c|}{$\begin{array}{c}\text { MRAC } \\
2 \text { Parám. }\end{array}$} & \multicolumn{2}{|c|}{\begin{tabular}{|c|} 
MRAC \\
1 Parám y Pred. \\
Smith
\end{tabular}} & \multicolumn{2}{|c|}{\begin{tabular}{|c|} 
MRAC \\
2 Parám y Pred. \\
Smith
\end{tabular}} & \multicolumn{2}{|c|}{$\begin{array}{c}\text { MRAC } \\
\text { PD }\end{array}$} \\
\hline & NRMSE & $R^{2}$ & NRMSE & $R^{2}$ & NRMSE & $\mathrm{R}^{2}$ & NRMSE & $R^{2}$ & NRMSE & $\mathrm{R}^{2}$ & NRMSE & $R^{2}$ \\
\hline 1 & 20.78 & 95.72 & 59.47 & 64.71 & 52.77 & 72.34 & 23.90 & 94.44 & 25.45 & 93.62 & 20.37 & 96.08 \\
\hline 2 & 14.72 & 97.87 & 25.52 & 93.66 & 38.10 & 85.54 & 28.16 & 92.34 & 28.66 & 91.84 & 17.93 & 96.92 \\
\hline 3 & 18.55 & 96.59 & 28.55 & 92.01 & 36.59 & 86.70 & 27.78 & 92.44 & 29.75 & 91.22 & 15.40 & 97.75 \\
\hline 4 & 20.88 & 95.67 & 32.32 & 89.68 & 30.79 & 90.73 & 24.94 & 94.04 & 24.11 & 94.27 & 17.48 & 97.09 \\
\hline 5 & 31.48 & 90.10 & 27.02 & 92.82 & 37.51 & 86.05 & 21.10 & 95.74 & 24.19 & 94.23 & 22.96 & 94.95 \\
\hline 6 & 49.60 & 75.45 & 22.95 & 94.82 & 46.86 & 78.20 & 22.48 & 95.17 & 29.56 & 91.38 & 19.08 & 96.53 \\
\hline 7 & 17.36 & 97.00 & 30.99 & 90.57 & 34.14 & 88.43 & 22.10 & 95.38 & 23.64 & 94.50 & 19.69 & 96.33 \\
\hline 8 & 44.04 & 80.61 & 40.38 & 83.90 & 38.38 & 85.43 & 29.31 & 91.86 & 30.14 & 91.04 & 16.87 & 97.26 \\
\hline 9 & 33.01 & 89.12 & 31.07 & 90.45 & 44.89 & 79.98 & 21.87 & 95.65 & 24.06 & 94.30 & 20.60 & 95.90 \\
\hline 10 & 35.95 & 87.11 & 46.57 & 78.36 & 60.03 & 64.16 & 26.48 & 93.18 & 29.84 & 91.26 & 16.44 & 97.43 \\
\hline$\overline{\mu_{x}}$ & 28.64 & 90.52 & 34.48 & 87.10 & 42.00 & 81.76 & 24.81 & 94.02 & 26.94 & 92.77 & 18.68 & 96.62 \\
\hline ICC & \multicolumn{2}{|c|}{91.87} & \multicolumn{2}{|c|}{95.83} & \multicolumn{2}{|c|}{95.67} & \multicolumn{2}{|c|}{98.41} & \multicolumn{2}{|c|}{99.32} & \multicolumn{2}{|c|}{99.50} \\
\hline
\end{tabular}

Tabla 3: Resultados del cálculo de NRMSE, $R^{2}$ e ICC para el eje Y con cada controlador. Señal Símica

\begin{tabular}{|c|c|c|c|c|c|c|c|c|c|c|c|c|}
\hline \multirow[t]{2}{*}{ Test } & \multicolumn{2}{|c|}{ Fuzzy } & \multicolumn{2}{|c|}{$\begin{array}{l}\text { MRAC } \\
\text { 1 Parám. }\end{array}$} & \multicolumn{2}{|c|}{$\begin{array}{c}\text { MRAC } \\
2 \text { Parám. }\end{array}$} & \multicolumn{2}{|c|}{$\begin{array}{c}\text { MRAC } \\
1 \text { Parám y PS }\end{array}$} & \multicolumn{2}{|c|}{$\begin{array}{c}\text { MRAC } \\
2 \text { Parám y PS }\end{array}$} & \multicolumn{2}{|c|}{$\begin{array}{l}\text { MRAC } \\
\text { PD }\end{array}$} \\
\hline & NRMSE & $\mathrm{R}^{2}$ & NRMSE & $\mathrm{R}^{2}$ & NRMSE & $\mathrm{R}^{2}$ & NRMSE & $R^{2}$ & NRMSE & $R^{2}$ & NRMSE & $\mathrm{R}^{2}$ \\
\hline 1 & 25.26 & 93.62 & 20.20 & 95.96 & 31.58 & 90.11 & 19.32 & 96.34 & 17.96 & 96.78 & 12.87 & 98.38 \\
\hline 2 & 11.52 & 98.67 & 34.25 & 88.28 & 46.30 & 78.92 & 20.31 & 95.97 & 19.66 & 96.16 & 13.61 & 98.19 \\
\hline 3 & 13.27 & 98.24 & 24.85 & 93.92 & 35.69 & 87.44 & 19.58 & 96.23 & 19.29 & 96.28 & 16.87 & 97.19 \\
\hline 4 & 88 & 96.05 & 26.54 & 93.10 & 39.35 & 84.76 & 23.09 & 94.72 & 15.31 & 97.65 & 18.71 & 96.56 \\
\hline 5 & 33.58 & 88.73 & 40.99 & 83.21 & 32.56 & 89.64 & 27.95 & 92.36 & 20.22 & 95.92 & 13.53 & 98.20 \\
\hline 6 & 31.53 & 90.07 & 22.92 & 94.76 & 33.14 & 89.33 & 41.63 & 82.76 & 15.52 & 97.59 & 14.19 & 98.03 \\
\hline 7 & 37.82 & 85.73 & 19.81 & 96.17 & 46.30 & 78.97 & 25.90 & 93.43 & 21.34 & 95.44 & 17.12 & 97.08 \\
\hline 8 & 11.57 & 98.66 & 20.95 & 95.70 & 51.94 & 73.20 & 19.95 & 96.09 & 19.80 & 96.08 & 16.29 & 97.36 \\
\hline 9 & 20.79 & 95.68 & 19.08 & 96.47 & 36.35 & 87.04 & 20.04 & 96.08 & 15.14 & 97.71 & 15.53 & 97.62 \\
\hline 10 & 36.29 & 86.83 & 59.82 & 64.22 & 36.04 & 87.22 & 22.58 & 94.96 & 22.63 & 94.89 & 13.67 & 98.17 \\
\hline$\overline{\mu_{y}}$ & 24.15 & 93.23 & 28.94 & 90.18 & 38.92 & 84.66 & 24.04 & 93.89 & 18.69 & 96.45 & 15.24 & 97.68 \\
\hline ICC & 94.1 & & 93.0 & & 98.5 & & 97. & & 99.4 & & 99. & \\
\hline
\end{tabular}

En las figuras 10 y 11 se presentan los resultados obtenidos en la generación de la señal sísmica "Imperial Valley" empleando el sistema de control MRAC-PD para los ejes X y Y respectivamente. En estas figuras se muestra el mejor y el peor resultado obtenido para cada eje, con lo que se puede comprobar el alto grado de repetitividad del sistema de control. En las figuras 12 y 13 se presenta la ampliación de algunas secciones de la figura 10 y 11 en los picos más representativos, con el fin de visualizar con mayor detalle la respuesta del controlador cuando requiere realizar desplazamientos máximos.

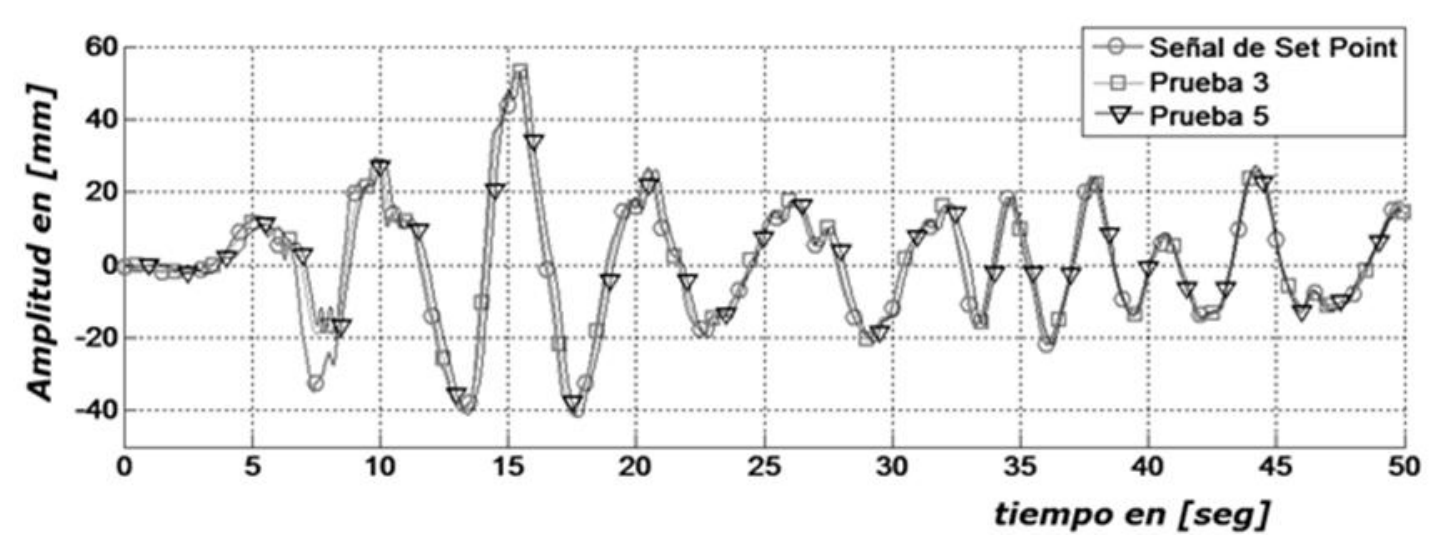

Fig. 10: Respuesta del control MRAC-PD ante el sismo Imperial Valley, eje X. 


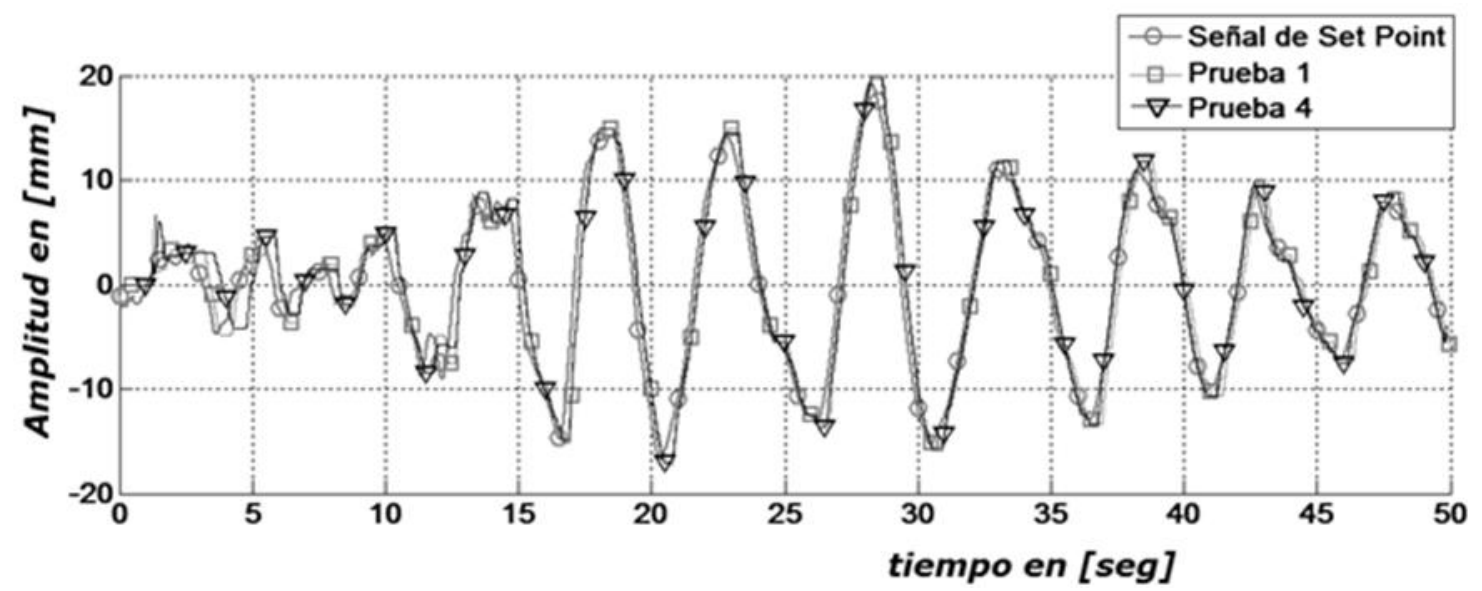

Fig. 11: Respuesta del control MRAC-PD ante el sismo Imperial Valley, eje Y.
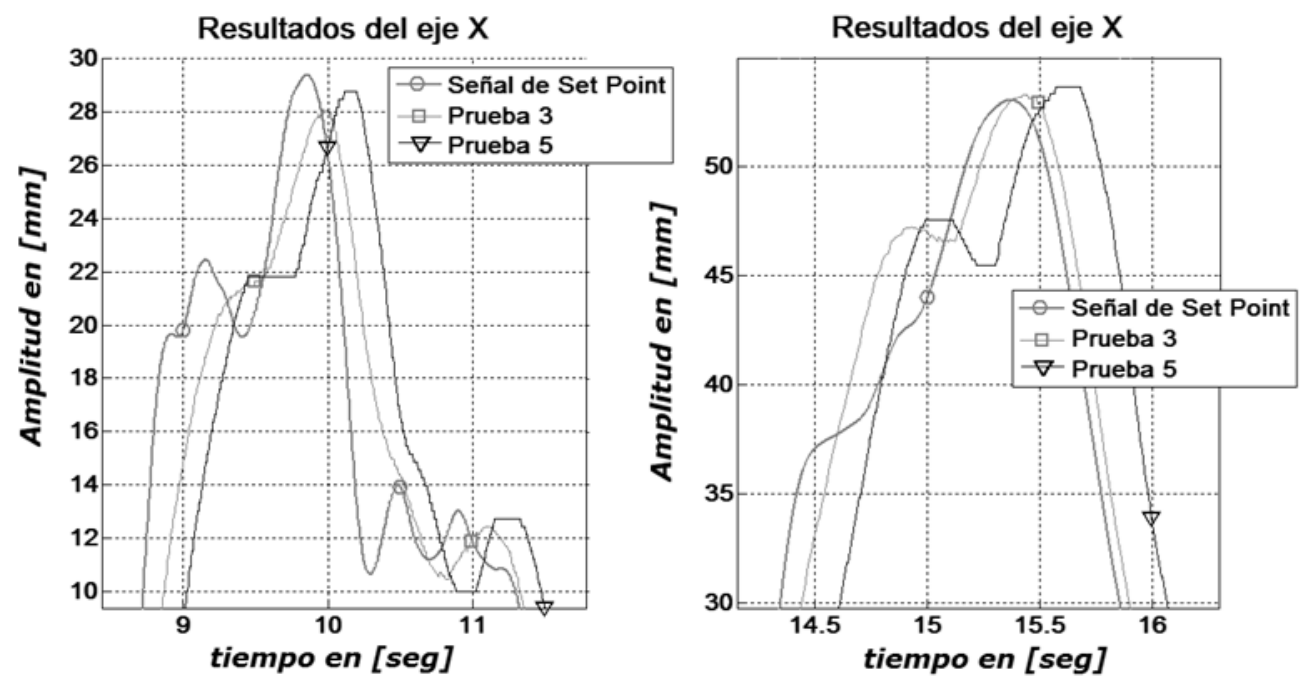

Fig. 12: Ampliación de la repuesta del controlador MRAC-PD en el eje $\mathrm{X}$ en los dos primeros picos representativos de la señal
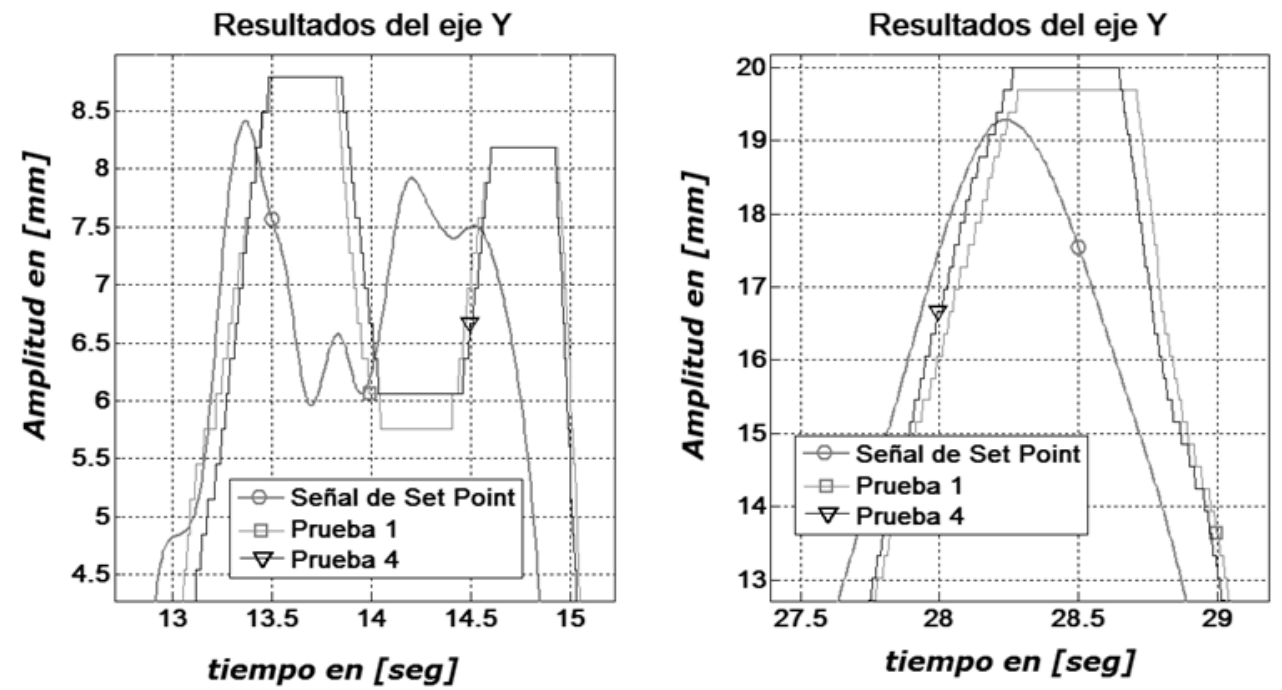

Fig. 13: Ampliación de la repuesta del controlador MRAC-PD en el eje $Y$ en dos de los picos más representativos de la señal

Por su parte, los resultados de los las señales senoidales se presentan en las tablas 4 y 5 , en las que a diferencia de las tablas 2 y 3 en las que se presentaban los resultados de cada experimento, solo se incluyen los resultados promedio de las métricas de error para cada frecuencia generada en la mesa vibratoria. 
Tabla 4: Resultados del cálculo de NRMSE, $R^{2}$ e ICC para el eje X con cada controlador. Señales Senoidales

\begin{tabular}{|c|c|c|c|c|c|c|c|c|c|c|c|c|c|c|c|c|c|}
\hline \multirow{2}{*}{$\begin{array}{l}\text { Frec. } \\
{[\mathrm{Hz}]}\end{array}$} & \multicolumn{3}{|c|}{ Euzzy } & \multicolumn{3}{|c|}{$\begin{array}{c}\text { MRAC } \\
\text { 1 Parámetro }\end{array}$} & \multicolumn{3}{|c|}{$\begin{array}{c}\text { MRAC } \\
2 \text { Parámetros }\end{array}$} & \multicolumn{3}{|c|}{$\begin{array}{c}\text { MRAC } \\
\text { 1 Parám. y PS }\end{array}$} & \multicolumn{3}{|c|}{$\begin{array}{c}\text { MRAC } \\
2 \text { Parám. y PS }\end{array}$} & \multicolumn{2}{|c|}{$\begin{array}{l}\text { MRAC } \\
\text { PD }\end{array}$} \\
\hline & IRMCE & $\mathrm{R}^{2}$ & C & ZMSE & $\mathrm{R}^{2}$ & IC & RMSE & $\mathrm{R}^{2}$ & ICC & RMSE & $\mathrm{R}^{2}$ & $1 \mathrm{ICO}$ & RMSE & $\mathrm{R}^{2}$ & - & & $\mathrm{R}^{2}$ \\
\hline 0.4 & & - & & & 88.7 & & & 98.7 & & & 99.1 & & & 99.2 & & & \\
\hline & & & & & 1 & & & D & & & & & & 2 & & & \\
\hline 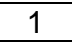 & & 9.0 & & & 98.7 & & & 98.7 & & & 99.0 & & & 99.2 & & & \\
\hline 2 & & 99.1 & & & 98.9 & & & 98.7 & & & 98.9 & & & 99.2 & & & \\
\hline 3 & 6.24 & 98.2 & 99.5 & 21 & 98.6 & 9.1 & 12 & 98.6 & 9 & 6.55 & 98.8 & 99. & 5. & 99.1 & 99. & 6.43 & 8. \\
\hline
\end{tabular}

Tabla 5: Resultados del cálculo de NRMSE, $R^{2}$ e ICC para el eje Y con cada controlador. Señales Senoidales

\begin{tabular}{|c|c|c|c|c|c|c|c|c|c|c|c|c|c|c|c|c|c|}
\hline \multirow{2}{*}{$\begin{array}{l}\text { Frec. } \\
{[\mathrm{Hz}]}\end{array}$} & \multicolumn{3}{|c|}{ Fuzzy } & \multicolumn{3}{|c|}{$\begin{array}{c}\text { MRAC } \\
\text { 1 Parámetro }\end{array}$} & \multicolumn{3}{|c|}{$\begin{array}{c}\text { MRAC } \\
2 \text { Parámetros }\end{array}$} & \multicolumn{3}{|c|}{$\begin{array}{c}\text { MRAC } \\
1 \text { Parám. y PS }\end{array}$} & \multicolumn{3}{|c|}{$\begin{array}{c}\text { MRAC } \\
2 \text { Parám. y PS }\end{array}$} & \multicolumn{2}{|c|}{$\begin{array}{l}\text { MRAC } \\
\text { PD }\end{array}$} \\
\hline & - & $i^{2}$ & & RMSE & $\mathrm{R}^{2}$ & ICC & NRMSE & $\mathrm{R}^{2}$ & ICC & NRMSE & $\mathrm{R}^{2}$ & ICC & NRMSE & $\mathrm{R}^{2}$ & ICC & ISE & $\mathrm{R}^{2}$ \\
\hline & & 9.2 & & & 99.1 & & & 99.1 & & & 99.1 & & & 99.1 & & & 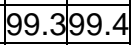 \\
\hline & & & & & & & & & & & & & & 0 & & & \\
\hline & & & & & & & & $0 .<$ & & & U & & & & & & \\
\hline 2 & & 9.1 & & & 99.1 & & & 99.2 & & & 99.2 & & & 9.1 & & & \\
\hline 3 & 5.78 & 98.8 & 99.5 & 9.78 & 98.9 & 99.4 & 9.80 & 98.7 & 99.4 & 6.11 & 98.8 & 99.5 & 6.17 & 99.0 & 99.5 & 5.98 & 98.999 \\
\hline
\end{tabular}

De los resultados de las tablas 4 y 5 se aprecia que el sistema de control Fuzzy presenta mejores resultados que los sistemas de control adaptativos, específicamente en la métrica de la raíz del error medio cuadrático normalizado. Esto se debe a que la salida de los controladores adaptativos dependen de la ganancia de adaptación $\gamma \mathrm{y}$, dado que el inicio de las señales senoidales es menor a $5 \mathrm{~mm}$, los parámetros de adaptación requieren del primer semiciclo de la señal senoidal para ajustarse e igualarse a la respuesta del modelo de referencia.

Este mismo efecto se aprecia en la figura 11, en la que la respuesta de la mesa en el eje $Y$ al inicio de la señal sísmica es lejana al setpoint, pero una vez la amplitud de la entrada supera los $5 \mathrm{~mm}$ el resultado del controlador MRAC se ajusta para seguir correctamente a la señal de referencia. Sin embargo, haciendo un análisis posterior de las salidas de todos los controladores, en el que se elimina el primer cuarto de periodo de la señal senoidal, los resultados de todos los controladores adaptativos se igualan y superan a los resultados del controlador Fuzzy. En la tabla 6 se presenta este análisis, en el que solo se muestra los resultados de la medida NRMSE para el eje X y el eje $\mathrm{Y}$. Los parámetros $R^{2}$ e ICC no se presentan ya que los resultados son cercanos a $100 \%$.

Tabla 6: Resultados del cálculo de NRMSE, para los ejes X y Y con cada controlador. Señales Senoidales eliminando el primer cuarto de periodo de la señal senoidal.

\begin{tabular}{|c|c|c|c|c|c|c|c|c|c|c|c|c|}
\hline \multirow{3}{*}{$\begin{array}{l}\text { Frec. } \\
{[\mathrm{Hz}]}\end{array}$} & \multicolumn{2}{|c|}{ FUZZY } & \multicolumn{2}{|c|}{$\begin{array}{c}\text { MRAC } \\
\text { 1 Parámetro }\end{array}$} & \multicolumn{2}{|c|}{$\begin{array}{c}\text { MRAC } \\
\text { 2 Parámetros }\end{array}$} & \multicolumn{2}{|c|}{$\begin{array}{c}\text { MRAC } \\
\text { 1 Parám. y PS }\end{array}$} & \multicolumn{2}{|c|}{$\begin{array}{c}\text { MRAC } \\
2 \text { Parám. y PS }\end{array}$} & \multicolumn{2}{|c|}{$\begin{array}{l}\text { MRAC } \\
\text { PD }\end{array}$} \\
\hline & \multicolumn{2}{|c|}{ NRMSE } & \multicolumn{2}{|c|}{ NRMSE } & \multicolumn{2}{|c|}{ NRMSE } & \multicolumn{2}{|c|}{ NRMSE } & \multicolumn{2}{|c|}{ NRMSE } & \multicolumn{2}{|c|}{ NRMSE } \\
\hline & Eje X & Eje $Y$ & Eje $X$ & Eje $Y$ & Eje $X$ & Eje $Y$ & Eje $X$ & Eje Y & Eje $X$ & Eje Y & Eje X & Eje Y \\
\hline 0.4 & 3.30 & 3.21 & 3.41 & 3.30 & 3.43 & 3.24 & 3.28 & 3.17 & 3.15 & 3.04 & 3.16 & 3.14 \\
\hline 0.7 & 3.28 & 3.25 & 3.45 & 3.28 & 3.44 & 3.37 & 3.33 & 3.20 & 3.11 & 3.12 & 3.14 & 3.23 \\
\hline 1 & 3.3 & 3.2 & 3.40 & 3.39 & 3.51 & 3.41 & 3.30 & 3.18 & 3.18 & 3.08 & 3.13 & 3.02 \\
\hline 2 & 3.31 & 3.24 & 3.39 & 3.42 & 3.47 & 3.32 & 3.29 & 3.11 & 3.20 & 3.29 & 3.14 & 3.09 \\
\hline 3 & 6.24 & 5.78 & 5.31 & 5.87 & 5.02 & 6.05 & 5.07 & 5.45 & 5.01 & 5.47 & 4.98 & 5.01 \\
\hline
\end{tabular}

En el análisis de respuesta a la entrada escalón se aprecia el efecto de la velocidad máxima que se puede generar con la mesa vibratoria, por lo que a su vez el ancho de banda de la misma se ve limitado a un máximo de $4 \mathrm{~Hz}$ con amplitudes pico a pico de solo $25 \mathrm{~mm}$. Este efecto también se vio en los resultados de la señal senoidal a $3 \mathrm{~Hz}$, en los que la salida no alcanza a desplazarse $40 \mathrm{~mm}$ pico a pico en las transiciones de la señal, razón por la que el error NRMSE es menor que en las frecuencias más bajas. En la tabla 7 se presentan los resultados de la respuesta promedio delos sistemas ante señales de entrada cuadradas con periodo de 10 segundos. En estos resultados se puede apreciar que aunque la repetividad de los experimentos es elevada, el error NRMSE y el coeficiente $R^{2}$ son bajos debido a que los controladores no pueden alcanzar el setpoint en menos de 1.36 segundos.

Otro aspecto a mencionar en los resultados de la tabla 7 es que aunque el controlador Fuzzy responde igual desde el inicio del experimento, este presenta oscilaciones antes de estabilizarse. En cuanto a los 
controladores MRAC, aunque estos tardan el primer segundo de las pruebas en adaptarse, la respuesta es más estable y rápida que el controlador Fuzzy, razón por la que los resultados fueron similares y en los últimos dos casos fueron mejores.

Tabla 7: Resultados promedio del cálculo de NRMSE, $R^{2}$ e ICC para los ejes $\mathrm{X}$ y $\mathrm{Y}$ con cada controlador.Señales Cuadradas

\begin{tabular}{|c|c|c|c|c|c|c|c|c|c|c|c|c|c|c|c|c|c|}
\hline \multirow[t]{2}{*}{ Ejes } & \multicolumn{3}{|c|}{ uzzy } & \multicolumn{3}{|c|}{$\begin{array}{c}\text { MRAC } \\
\text { 1 Parámetro }\end{array}$} & \multicolumn{3}{|c|}{$\begin{array}{c}\text { MRAC } \\
2 \text { Parámetros }\end{array}$} & \multicolumn{3}{|c|}{$\begin{array}{c}\text { MRAC } \\
\text { 1 Parám. y PS }\end{array}$} & \multicolumn{3}{|c|}{$\begin{array}{c}\text { MRAC } \\
2 \text { Parám. y PS }\end{array}$} & \multicolumn{2}{|c|}{$\begin{array}{l}\text { MRAC } \\
\text { PD }\end{array}$} \\
\hline & $\Lambda$ & $\mathrm{R}^{2}$ & C & RMSE & $R^{2}$ & ICC & USE & $\mathrm{R}^{2}$ & ICC & MSE & $\mathrm{R}^{2}$ & ICC & MSE & $\mathrm{R}^{2}$ & ICC & $\mathrm{E}$ & $R^{2}$ \\
\hline 51 & .34 & & 98.6 & 57 & 93.1 & 99.1 & .44 & 93.4 & 99.2 & 86 & 94.1 & 99.2 & 98 & 94.5 & 99.2 & 9.28 & 35. \\
\hline je $Y$ & 32 & 3.0 & 98.7 & 33 & 92.8 & 99.2 & 21 & 93.6 & 9.2 & 77 & 93.9 & 99.1 & 40 & 95.2 & 99.3 & 9.09 & טjן \\
\hline
\end{tabular}

De los resultados de estas tablas se aprecia que el sistema de control que presentó los mejores resultados, basados en los tres parámetros de medición utilizados, fue el control adaptativo MRAC PD seguido por el sistema de control MRAC con dos parámetros y predictor Smith, mostrando mayor estabilidad en la repetitividad de las pruebas. En parte la varianza entre las pruebas con los otros sistemas de control se debe a que no responde suficientemente rápido ante variaciones en los parámetros que afectan el desempeño de la planta, como el rozamiento de los materiales sobre los cuales se deslizan los ejes, que se aprecian en las figuras 2 y 3 , o en especial al retardo ocasionado por el sistema de cadenas que transmite la energía rotacional de los motores.

\section{CONCLUSIONES}

La implementación de los diferentes tipos de controladores para el sistema basado en la mesa vibratoria permitió validar el comportamiento adecuado de los sistemas de control adaptativo por modelo de referencia cuando son usados en procesos en los que no se conoce con exactitud el modelo matemático del proceso a controlar, inclusive para el caso de un sistema de control Fuzzy el cual permite ciertos grados de incertidumbre. De esta manera, en este trabajo se analizó el comportamiento se los seis diferentes controladores ante diferentes señales de entrada, aun cuando la finalidad de la mesa vibratoria es para generar señales sísmicas, en los que se obtuvieron resultados adecuados para todos los controladores, en los que el mejor resultado se presentó en el controlador MRAC-PD.

Otro aspecto importante que se obtiene de los resultados es que el acople del predictor Smith mejoró el comportamiento de los sistemas de control implementados. A su vez se demostró que los errores ocasionados por los retardos de la planta, debido a las cadenas del mecanismo de movimiento de cada eje, se podían minimizar analizando a la planta como una sin retardo y en cascada con un tiempo muerto, lo cual fue aplicado en la obtención de los modelos de referencia de cada uno de los controladores.

Finalmente, un aspecto importante que se obtuvo en todos los controladores adaptativos proporcionales implementados es, que los parámetros de adaptación se ajustan rápidamente en el instante que la señal de entrada supera los $5 \mathrm{~mm}$. Esto se aprecia gráficamente al inicio de la figura 11, y en los resultados de las señales de prueba senoidales, en las que el error es mayor comparado con la respuesta de los controladores Fuzzy dado que la respuesta inicial se aleja de la esperada hasta que los parámetros de adaptación se estabilizan. Por otra parte, el controlador MRAC-PD mejora este comportamiento debido a que la parte derivativa del controlador genera adecuadamente una acción e control ante las variaciones de la señal del error, lo cual se aprecia en los resultados con señales cuadradas, dado que la razón de cambio del error es superior que en los otros casos analizados.

\section{REFERENCIAS}

Aliane N., J. Fernández, A. Martínez y J. Ortiz; Un Laboratorio de Ingeniería de Control Basado En Internet, Información Tecnológica 18 (6), 19-26 (2007).

Chen J., X. Zhang, P. Tan y F. Zhou; Application of the Optimal Input Design in Shaking Table Experimental System, In Robotics, Automation and Mechatronics, 2008 IEEE Conference On, 243-247, IEEE (2008).

Duraisamy R. y S. Dakshinamurthy; An Adaptive Optimisation Scheme for Controlling Air Flow Process with Satisfactory Transient Performance, Maejo International Journal of Science and Technology 4 (02), 221-234 (2010). 
Esparza C., R. Núñez y F. González; Model Reference Adaptive Position Controller with Smith Predictor for a Shaking-table in Two Axes, Advances in Computational Intelligence 7630, 271-282 (2013).

Esparza C., R. Núñez y M. Pedraza; Control Fuzzy Para La Generación de Señales Sísmicas en una Mesa Vibradora, con Desplazamiento de sus Ejes Basado en un Mecanismo Biela-manivela, Revista Especializada En Sistemas Informáticos y Electrónicos de Telecomunicaciones RESET-UTS 6 (1), 18-27 (2011).

Frýza T. y S. Hanus; Video Signals Transparency in Consequence of 3d-dct Transform, In Radioelektronika 2003 Conference Proceedings, 127-130 (2003).

García-Nieto S., M. Martínez, Á. Llosá y J. Sanchis; Estrategias de Enseñanza a Distancia Sobre Control No-Lineal Aplicada Al Péndulo Invertido, Información Tecnológica 18 (5), 85-98 (2007).

loacnou P.; "Chapter 54. Model Reference Adaptive Control", In The Control Handbook, 847-858. CRC press - IEEE press (2000).

Landau I., R. Lozano, M. M'Saad y A. Karimi; "Adaptive Control: Algorithms, Analysis and Applications", Second Edition, Springer (2011).

Mariño, P., V. Pastoriza, M. Santamaría y E. Martínez; Sistema Borroso de Inspección del Rebarnizado de Tapas en la industria Metalgráfica, Información Tecnológica 17 (4), 17-22 (2006).

McGraw K. y S. P. Wong; Forming Inferences About Some Intraclass Correlation Coefficients, Psychological Methods 1 (1), 3-46 (1996).

Montgomery D. C. y G. Runger; Applied Statistics and Probability for Engineers, Third Edition, John Wiley \& Sons (2007).

Normey-Rico J. y E. Camacho; Predicción Para Control: Una Panorámica Del Control de Procesos Con Retardo, Revista Iberoamericana de Automática e Informática Industrial RIAI 3 (4), 5-25 (2006).

PayáL., O. Reinoso, A. Gil y L. Jiménez; Plataforma Distribuida para la Realización de Prácticas de Robótica Móvil a través de Internet, Información Tecnológica 18 (6), 27-38 (2007).

Rodríguez F. y M. J. López; "Control Adaptativo y Robusto", Secretariado de Publicaciones de la Universidad de Sevilla (1996).

Seki K. y otros cuatro autores; Adaptive Feedforward Compensation for Reaction Force with Nonlinear Specimen in Shaking Tables, Conference On Mechatronics 2009 - ICM 2009, IEEE International, 1-6, IEEE (2009).

Swarnkar P., S. Jain y R. K. Nema; Effect of Adaptation Gain on System Performance for Model Reference Adaptive Control Scheme Using MIT Rule, In World Academy of Science, Engineering and Technology, 621-626 (2010).

Swarnkar P., S. Jain y R. K. Nema; Effect of Adaptation Gain in Model Reference Adaptive Controlled Second Order System, ETASR - Engineering, Technology \& Applied Science Research 1 (3), 70-75 (2011).

Tu J. W., S. J. Jiang y D. P. Stoten; The Seismic Response Reduction by Using Model Reference Adaptive Control Algorithm, In Mechanic Automation and Control Engineering (MACE), 2010 International Conference On, 1215-1218, IEEE (2010).

Yao J., W. Fu, S. Hu y S. Liu; XPC-based Real Time Control System for an Electro-hydraulic Servo Shaking Table, In 8th World Congress on Intelligent Control and Automation (WCICA), 2010, 2026-2030, IEEE (2010). 